\title{
Radiographic Imaging in Autosomal Dominant Polycystic Kidney Disease: A Claims Analysis
}

\author{
Myrlene Sanon Aigbogun \\ Robert A Stellhorn' \\ Christina S Pao (D) \\ Stephen L Seliger ${ }^{2}$
}

'Otsuka Pharmaceutical Development \& Commercialization, Inc, Princeton, NJ, USA; ${ }^{2}$ University of Maryland School of Medicine, Baltimore, MD, USA
Correspondence: Stephen L Seliger University of Maryland School of

Medicine, 10 N. Greene Street,

Baltimore, MD, 2I20I, USA

Tel +410 605-7000 x523I

$\mathrm{Fax}+410328-5685$

Email sseliger@som.umaryland.edu
Background: Progression of autosomal dominant polycystic kidney disease (ADPKD) is highly variable, with some patients progressing rapidly to end-stage renal disease (ESRD). Abdominal imaging is an important modality for verifying diagnosis in patients at risk for rapidly progressing ADPKD, targeting them for early treatment that could slow onset of ESRD. Published literature is limited on the real-world abdominal imaging utilization patterns in ADPKD.

Methods: A retrospective healthcare administrative claims analysis examining abdominal imaging scans occurring from January 1, 2014, through June 30, 2017, was conducted using the IBM MarketScan ${ }^{B}$ commercial and Medicare supplemental databases. Patients in the United States who were at least 18 years old and had at least 1 inpatient claim or 2 outpatient claims (with different dates of service) with an ADPKD diagnosis code, as defined by the International Classification of Diseases, Ninth Revision, Clinical Modification (ICD-9-CM codes 753.12 [polycystic kidney, unspecified type] and 753.13 [polycystic kidney, autosomal dominant] and/or Tenth Revision (ICD-10-CM codes Q61.2 [polycystic kidney, adult type] and Q61.3 [polycystic kidney, unspecified]) were included.

Results: Of the 4637 patients with ADPKD (mean age, 51.2 years [SD $=15.52]$ ), 59\% had $\geq 1$ abdominal imaging scan. Of these patients, $46 \%$ had $\geq 1$ computed tomography (CT) scan, $25 \%$ had $\geq 1$ ultrasound, $10 \%$ had $\geq 1$ magnetic resonance imaging scan. Among the 1754 patients (38\%) with chronic kidney disease (CKD) stage information, CT imaging was more frequent in later stages (31\% stage 1 versus $68 \%$ stage 5$)$. The proportion of patients undergoing at least $1 \mathrm{CT}$ or MRI scan increased with disease severity $(37 \%$ in stage 1 , $42 \%$ in stage $2,48 \%$ in stage $3,56 \%$ in stage 4 , and $71 \%$ in stage 5 ).

Conclusion: Results of this analysis support the need for further investigation into abdominal imaging utilization in managing patients with ADPKD. Future research could clarify barriers and increase access to imaging, which has the potential to inform risk stratification, help patients delay dialysis or transplantation associated with ESRD, and help health systems avoid the costs associated with ESRD.

Keywords: polycystic kidney, autosomal dominant, kidney diseases, cystic, MRI, CT scan, ultrasound

\section{Introduction}

Autosomal dominant polycystic kidney disease (ADPKD) is one of the most common inherited kidney diseases. ${ }^{1,2}$ ADPKD is the fourth leading cause of end-stage renal disease (ESRD) in the United States, accounting for approximately 5\% of all cases. ${ }^{3}$ The point prevalence of ADPKD in the United States has been estimated to be $<1$ case per 2000 individuals, a rate translating to approximately 140,000 patients in most recent estimates. ${ }^{4}$ 
Many patients with ADPKD remain symptom-free for decades. Most patients, however, eventually experience a steady decline in glomerular filtration rate (GFR) even if they remain asymptomatic or minimally asymptomatic. ${ }^{1,5,6}$ By age 50 , more than half of patients with ADPKD will have developed ESRD, requiring chronic dialysis or kidney transplant. ${ }^{4}$

However, the rate of disease progression varies markedly between patients. The National Institutes of Healthsponsored Consortium for Radiologic Imaging Studies of Polycystic Kidney Disease (CRISP) determined that kidney growth (as measured by change in height-adjusted total kidney volume [htTKV]) predicts future GFR decline. $^{6-8}$ Together with age and estimated GFR (eGFR), TKV is considered by the US Food and Drug Administration (FDA) and European Medicines Agency as a prognostic biomarker of subsequent renal function decline. ${ }^{9}$ Validated risk prediction models incorporating $\mathrm{TKV}$, height, age, and current kidney function can predict future risk of ESRD. ${ }^{9}$

Two pivotal clinical trials demonstrated that tolvaptan, a vasopressin type-2 receptor antagonist, can slow disease progression in adults with rapidly progressing ADPKD. The Tolvaptan Efficacy and Safety in Management of Autosomal Dominant Polycystic Kidney Disease and Its Outcomes (TEMPO) 3:4 trial (NCT00428948) showed that in subjects with early ADPKD (creatinine clearance $>60 \mathrm{~mL} / \mathrm{min}$ ), tolvaptan reduced kidney growth by $45 \%$ and eGFR decline by $26 \%$ over 3 years. ${ }^{10}$ The Replicating Evidence of Preserved Renal Function: An Investigation of Tolvaptan Safety and Efficacy in ADPKD (REPRISE) trial (NCT02160145) found that in subjects with advanced ADPKD (eGFR 25-65 $\mathrm{mL} / \mathrm{min}$ per $1.73 \mathrm{~m}^{2}$ ), eGFR decline was reduced by $35 \%$ with tolvaptan therapy over a 1-year period. ${ }^{11}$ These results supported the April 2018 FDA approval of tolvaptan to slow kidney function decline in adults at risk of rapidly progressing ADPKD. ${ }^{12}$

However, appropriate risk stratification is essential to select patients for potential tolvaptan therapy. Several investigators have suggested practical approaches to this risk stratification. ${ }^{13}$ For example, Chebib et al recommended the Mayo imaging classification, in which tolvaptan use would be considered for patients in class IC, ID, or IE. ${ }^{14}$ The Mayo approach uses radiographic imaging data obtained from ultrasound, computed tomography (CT), or magnetic resonance imaging (MRI) scans to measure TKV. ${ }^{7}$ Chebib et al noted a preference for CT or MRI. ${ }^{14}$ Although ultrasonography is relatively low in cost and does not expose patients to radiation, it is less precise than the other methods and ineffective for assessing small cysts $(<1 \mathrm{~cm})$. MRI is the most sensitive and accurate imaging tool for measuring TKV and is as accurate as CT in visualizing small renal cysts $(<1 \mathrm{~cm})$ without the potential radiation exposure associated with CT scans. ${ }^{6,15}$ Image-based criteria and TKV data provided by these imaging tools allow for more-accurate diagnoses and practical risk stratification to identify patients most likely to benefit from effective therapy. ${ }^{7}$

Differentiating patients with rapidly progressing ADPKD from those with a more indolent condition could help improve disease management, ensuring that those patients who could benefit the most from early treatment receive such treatment, potentially delaying the onset of ESRD in this subgroup. However, little research has been conducted on the use of radiographic imaging scans in patients with ADPKD in a real-world clinical practice environment. To better characterize how imaging scans are currently used to evaluate patients with ADPKD in real-world settings, this study analyzed medical claims data to determine how imaging utilization patterns varied across disease severity and treatment settings.

\section{Materials and Methods}

This was a retrospective analysis of administrative medical claims data. All data were fully de-identified to protect patient confidentiality (eg, no names, addresses, social security or medical record numbers, or other obvious identifiers); informed consent was not required. This study is classified as non-human subject research given that all data were fully de-identified and could not be linked to identifiable information.

\section{Data Source}

Patients with ADPKD were identified using medical claims data from the IBM MarketScan ${ }^{\circledR}$ commercial and Medicare supplemental databases. These databases compile inpatient, outpatient, emergency department (ED), laboratory, radiology, and pharmacy claims data from approximately 43.6 million Medicare supplemental and commercial insurance beneficiaries in the United States. ${ }^{16}$ Claims data were used to identify baseline demographic and comorbid conditions, baseline disease severity, and imaging scan events. 


\section{Patient Population}

For study inclusion, patients must have had at least one inpatient claim or two outpatient claims (with different dates of service) from January 1, 2014, through June 30, 2017, with an ADPKD diagnosis code, as defined by the International Classification of Diseases, Ninth Revision, Clinical Modification (ICD-9-CM codes 753.12 [polycystic kidney, unspecified type] and 753.13 [polycystic kidney, autosomal dominant]) and/or Tenth Revision (ICD-10-CM codes Q61.2 [polycystic kidney, adult type] and Q61.3 [polycystic kidney, unspecified]); eligible diagnosis codes could be listed in any position on the medical claim.

An individualized "index date" was designated based on each patient's earliest claim date with an ADPKD diagnosis code. All eligible patients were also required to be at least 18 years old as of their index date and had to have been continuously enrolled with the same health plan for at least 6 months before and 6 months after their index date. Patients with a prior kidney transplant $(n=511)$ were not excluded. An individualized "study observation period" was defined as the period of continuous health plan enrollment within the study period (January 1, 2014, through June 30, 2017) inclusive of each patient's individualized index date. Lack of available data prevented a longer study time horizon.

\section{Study Measures}

Descriptive analyses of scan utilization patterns were supplemented with patient demographics, baseline comorbid conditions, chronic kidney disease (CKD) staging, and health care delivery settings.

\section{Patient Demographics}

Patient sex, age as of index date (grouped as 18-24, 25-34, 35-44, 45-54, 55-64, 65-74, 75-84, 85-94, 95+ years), and geographic region throughout the United States (Northeast, North Central, South, West) were captured.

\section{CKD Staging at Baseline}

Each patient's baseline CKD stage was determined using ICD-9-CM or ICD-10-CM diagnostic codes from inpatient and outpatient medical claims with dates of service before and including each patient's index date. In cases in which the patient had multiple diagnosis codes for different CKD stages, patients were assigned to their highest documented CKD stage. CKD stages were defined by ICD-9-CM and ICD-10-CM codes as stage 1 (585.1, N18.1, respectively), stage 2 (585.2, N18.2), stage 3 (585.3, N18.3), stage 4 (585.4, N18.4), and stage 5 (585.5, 585.6, N18.5, N18.6). ${ }^{17}$

\section{Baseline Comorbid Conditions}

The presence of common comorbid conditions at baseline was assessed for each patient using diagnosis codes from medical claims with dates of service within the 6 months before and including each patient's index date. The Quan ICD-9-CM and ICD-10 coding algorithm was used to identify 17 unique comorbid conditions. ${ }^{18}$ A Charlson comorbidity index score of $0,1,2,3$, or $4+$ was determined for each patient to reflect their predicted risk of 1-year mortality based on a range of comorbid conditions. ${ }^{19}$

\section{Health Care Setting}

As health care (including diagnostic imaging) utilization patterns may vary by treatment setting, all patients' radiographic imaging scan events that occurred after each patient's index date and within their study observation period were categorized by both health care setting (inpatient, outpatient) and by whether that episode originated in the ED. "EDInpatient ED" visits are ED visits that resulted in a hospital admission, whereas "ED-only" visits were ED visits that did not lead to an inpatient hospitalization. The IBM MarketScan claims data include a "service category" variable indicating whether a claim is from an ED visit; if this ED service category was on an inpatient claim, the inpatient hospitalization originated from an ED visit; otherwise, the visit was considered to be an outpatient visit.

\section{Imaging Scan Utilization}

The number and percentage of patients with ADPKD who underwent each specific type of radiographic imaging scan procedure during the observation period were captured using current procedural terminology, fourth edition (CPT-4) codes. Table 1 lists relevant CPT-4 codes by type of scan (CT, MRI, and ultrasound).

\section{Statistical Analysis}

Both descriptive and inferential statistics were used. Mean and standard deviation were calculated for all continuous measures, while the number and percentage of patients were calculated for categorical measures. Chi-squared tests were conducted to test the significance of differences across CKD stages and by health care setting (significance level $p<0.05$ ). 
Table I CPT-4 Codes for Radiographic Imaging Scans

\begin{tabular}{|c|c|c|}
\hline $\begin{array}{l}\text { Imaging } \\
\text { Scan Type }\end{array}$ & $\begin{array}{l}\text { CPT-4 } \\
\text { Code }\end{array}$ & Procedure \\
\hline $\begin{array}{l}\text { Abdominal } \\
\text { MRI }\end{array}$ & $\begin{array}{l}74181 \\
74182 \\
74183 \\
74185\end{array}$ & $\begin{array}{l}\text { MRI abdomen w/o contrast } \\
\text { MRI abdomen w/contrast } \\
\text { MRI abdomen w/o and w/contrast } \\
\text { MRI abdomen }\end{array}$ \\
\hline $\begin{array}{l}\text { Abdominal } \\
\text { CT }\end{array}$ & $\begin{array}{l}74150 \\
74160 \\
74170 \\
74174 \\
74175 \\
74176 \\
74177 \\
74178\end{array}$ & $\begin{array}{l}\mathrm{CT} \text { abdomen w/o contrast } \\
\mathrm{CT} \text { abdomen w/contrast } \\
\mathrm{CT} \text { abdomen w/o and w/contrast } \\
\mathrm{CT} \text { angio abdomen and pelvis contrast } \\
\text { w/and w/o contrast } \\
\mathrm{CT} \text { angio abdomen with contrast/ } \\
\text { noncontrast } \\
\mathrm{CT} \text { abdomen and pelvis w/o contrast } \\
\mathrm{CT} \text { abdomen and pelvis w/contrast } \\
\mathrm{CT} \text { abdomen and pelvis w/o contrast I } \\
\text { or both body regions }\end{array}$ \\
\hline $\begin{array}{l}\text { Abdominal } \\
\text { ultrasound }\end{array}$ & $\begin{array}{l}76700 \\
76705\end{array}$ & $\begin{array}{l}\text { U/S abdomen complete } \\
\mathrm{U} / \mathrm{S} \text { abdomen }\end{array}$ \\
\hline
\end{tabular}

Abbreviations: angio, angiography; CPT-4, current procedural terminology, fourth edition; CT, computed tomography; MRI, magnetic resonance imaging; U/S, ultrasound; w/, with; w/o, without.

\section{Results}

\section{Selection of Study Population}

A total of 14,809 patients met the ADPKD diagnostic criteria during the selection ascertainment period. Among these, 4905 patients had continuous enrollment, and 4637 patients were adults at the time of the initial ADPKD diagnosis (index date; Figure 1).

\section{Baseline Patient Demographics and Clinical Characteristics Patient Demographics}

Patients' mean age at baseline was 51 years (more than half being $<55$ years old), with slightly more women $(52 \%)$ than men (Table 2). Forty-two percent of patients lived in the South; no other geographic region (Northeast, North Central, West) contributed more than $25 \%$ to the total study population. Patients had a mean (standard deviation [SD]) length of follow-up from index date of 21.3 (8.8) months. Forty-eight percent of ADPKD patients with an ADPKD claim were under the care of a nephrologist.

\section{Comorbid Conditions}

As shown in Table 2, hypertension was the most prevalent comorbid condition, with $64 \%$ of patients having a hypertension diagnosis code within the 6 months before their index date. A history of kidney stones was indicated for $8 \%$ of the patients and urinary tract infection for $11 \%$. Diabetes and dyslipidemia were present in $13 \%$ and $14 \%$ of the patients, respectively. The following conditions were diagnosed in $<10 \%$ of patients: chronic pulmonary disease $(8 \%)$, mood disorder (7\%), anxiety (7\%), depression $(7 \%)$, sprains $(6 \%)$, and cancer/malignancy $(5 \%)$. The

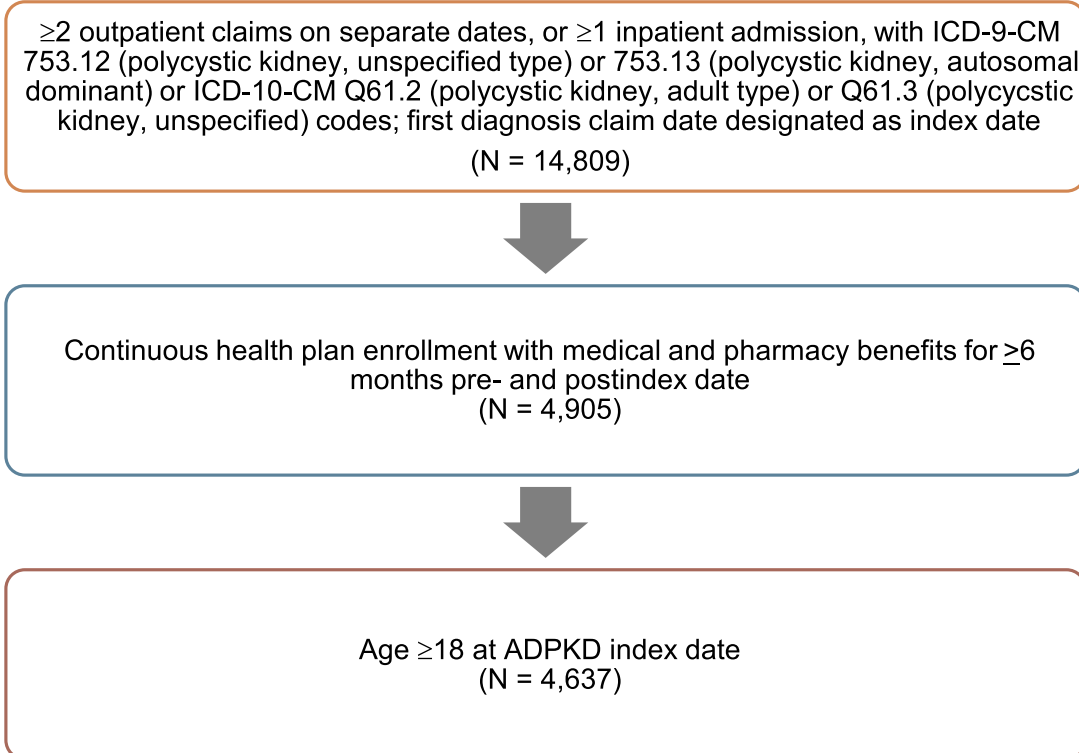

Figure I Selection of ADPKD analysis sample.

Abbreviations: ADPKD, autosomal dominant polycystic kidney disease; ICD-9-CM, International Classification of Diseases, Ninth Revision, Clinical Modification; ICD-I0CM, International Classification of Diseases, Tenth Revision, Clinical Modification. 
Table 2 Baseline Characteristics of Patients with ADPKD

\begin{tabular}{|c|c|}
\hline Characteristic & $\begin{array}{l}\text { All Patients } \\
(\mathrm{N}=4637)\end{array}$ \\
\hline Age, mean (SD), y & $51(15.5)$ \\
\hline \multicolumn{2}{|l|}{ Age, range, \% } \\
\hline $18-24$ & 6 \\
\hline $25-34$ & 10 \\
\hline $35-44$ & 17 \\
\hline $45-54$ & 24 \\
\hline $55-64$ & 26 \\
\hline $65-74$ & 10 \\
\hline $75+$ & 7 \\
\hline \multicolumn{2}{|l|}{ Sex, \% } \\
\hline Female & 52 \\
\hline Male & 48 \\
\hline \multicolumn{2}{|l|}{ Geographic region in the United States, \% } \\
\hline Northeast & 20 \\
\hline North Central & 23 \\
\hline South & 42 \\
\hline West & $15 \%$ \\
\hline Length of follow-up from index date, mean (SD), mo & $21.29(8.84)$ \\
\hline \multicolumn{2}{|l|}{ Comorbid conditions, \% } \\
\hline Hypertension & 64 \\
\hline Dyslipidemia & 14 \\
\hline Diabetes & 13 \\
\hline Urinary tract infection & II \\
\hline Chronic pulmonary disease & 8 \\
\hline Kidney stone & 8 \\
\hline Mood disorder & 7 \\
\hline Anxiety & 7 \\
\hline Depression & 7 \\
\hline Sprains & 6 \\
\hline Cancer/malignancy & 5 \\
\hline Charlson comorbidity index, mean (SD) & $1.44(1.73)$ \\
\hline \multicolumn{2}{|l|}{ Baseline CKD stage ( $N=1754), \%$} \\
\hline CKD stage I & 7 \\
\hline CKD stage 2 & 12 \\
\hline CKD stage 3 & 38 \\
\hline CKD stage 4 & 14 \\
\hline CKD stage 5 & 29 \\
\hline
\end{tabular}

Abbreviations: ADPKD, autosomal dominant polycystic kidney disease; CKD, chronic kidney, disease; mo, month; SD, standard deviation; $y$, year.

mean (SD) Charlson comorbidity index score was 1.44 (1.73).

\section{CKD Severity}

A diagnosis code indicating specific CKD stage was present in $38 \%$ of the patients in the analysis $(n=1754)$. More than two-thirds were categorized as either CKD stage 3 or stage 5 (Table 2), while the remaining patients were categorized as CKD stage 1,2 , or 4 . Laboratory data were not available as part of this analysis; these data would have been helpful in determining CKD staging for patients.

\section{Utilization of Imaging Scans}

Of the 4637 patients in the primary analysis cohort, 2717 (59\%) had at least 1 abdominal imaging scan throughout the observation period (Table 3). During this period, 1151 (25\%) had at least 1 ultrasound, $2146(46 \%)$ had at least 1 CT scan, and $451(10 \%)$ had at least 1 MRI scan. A total of 1920 patients (41\%) had no scan during the observation period.

Of patients who underwent abdominal CTs $(n=2146)$, $77 \%$ underwent CTs without contrast, 36\% CTs with contrast, and $10 \% \mathrm{CTs}$ with/without contrast. The percentage of CT scans with contrast was higher in stages 1-3, possibly because contrast studies are minimal in advancedstage patients whereas CT scans in earlier-stage patients can be used to evaluate solid tumor or infection. However, given the low number of patients (38\%) identified out of the full population with CKD staging information, it is difficult to draw conclusions from these percentages.

\section{Imaging Utilization by Disease Severity}

Across all CKD stages, CT scans were by far the most widely utilized abdominal imaging modality compared with MRI or ultrasound during patients' study observation periods (Figure 2). The proportion of patients undergoing at least $1 \mathrm{MRI}$ or CT scan varied by stage and increased with disease severity (37\% in stage $1,42 \%$ in stage $2,48 \%$ in stage 3,56\% in stage 4 , and $71 \%$ in stage 5 ). A Cochran-Armitage test was performed to assess the trend for increasing likelihood of imaging with increasing CKD stage, and the results showed that utilization of scans significantly increased with higher (or more advanced) CKD stages ( $\mathrm{z}$ statistic -9.55 , two-sided $P<0.001$ ). A significant association was also detected between ultrasound frequency and CKD stage $(p<0.0001)$. However, no significant association was found between frequency of MRI and CKD stage. The proportion of patients who underwent at least 1 MRI scan never exceeded $15 \%$ in any CKD stage and did not vary substantially by stage.

\section{Imaging Utilization by Health Care Setting}

Imaging utilization patterns varied not only by CKD stage but also by health care setting (Table 3 ). All but 1 of the 
Table 3 Utilization of Imaging Scans in ADPKD Patients by Health Care Setting

\begin{tabular}{|l|c|c|c|c|c|}
\hline & $\begin{array}{c}\text { Any Setting } \\
\mathbf{( N = 4 6 3 7 )}\end{array}$ & $\begin{array}{c}\text { Outpatient } \\
(\mathbf{n}=\mathbf{4 6 3 6})\end{array}$ & $\begin{array}{c}\text { ED-Only } \\
\mathbf{( n = 2 4 5 3 )}\end{array}$ & $\begin{array}{c}\text { Inpatient } \\
(\mathbf{n}=\mathbf{I 7} \text { I6) }\end{array}$ & $\begin{array}{c}\text { ED } \rightarrow \text { Inpatient ED } \\
(\mathbf{n}=\mathbf{I} \text { (87) }\end{array}$ \\
\hline No. (\%) of patients scanned ${ }^{\mathrm{a}}$ & $2717(58.6)$ & $2356(50.8)$ & $680(27.7)$ & $516(30.1)$ & $150(12.6)$ \\
\hline Abdominal CT & $2146(46.3)$ & $1723(37.2)$ & $643(26.2)$ & $437(25.5)$ & $142(12.0)$ \\
\hline Abdominal MRI & $45 I(9.7)$ & $415(9.0)$ & $6(0.2)$ & $46(2.7)$ & $0(0.0)$ \\
\hline Ultrasound & $1151(24.8)$ & $993(21.4)$ & $94(3.8)$ & $166(9.7)$ & $24(2.0)$ \\
\hline
\end{tabular}

Note: ${ }^{a}$ Individual patients may have more than one type of scan within a single health care setting.

Abbreviations: $\mathrm{CT}$, computed tomography; ED, emergency department; MRI, magnetic resonance imaging.

4637 patients in the analysis had been seen at least once for ADPKD in an outpatient setting during their study observation period. Of these, $51 \%$ received at least 1 imaging scan during an outpatient visit. More than a third of these patients who underwent scanning during an outpatient visit received at least $1 \mathrm{CT}$ scan. Less than $10 \%$ of the outpatients underwent an MRI scan.

More than half of all patients in the analysis $(n=2453)$ had at least one ADPKD-related ED-only visit that did not lead to an inpatient hospitalization ("ED-only"), which may be indicative of a more serious kidney event (Table 3). Despite their outpatient utilization having stemmed from emergency care, only a quarter of these patients received an imaging scan during these visits. For patients who were scanned during an ED-only visit, nearly all received a CT scan. Use of MRI scans and ultrasounds in this health care setting was negligible.
As scans during hospitalizations may represent moresevere cases, scans during an inpatient hospitalization were examined for two nested subgroups of hospitalized patients: those who were hospitalized via an ED visit and the larger subgroup of those who were hospitalized regardless of whether that hospitalization originated from an ED visit. Roughly a third of patients in the analysis $(n=1716)$ had an inpatient hospitalization (admitted via ED or not) during their study observation period. Of these hospitalized patients, nearly a third received an imaging scan during their hospitalization. As was similar to patients treated in an outpatient setting, CT scans were the most frequently used imaging modality.

A total of 1187 patients in the analysis $(26 \%)$ were admitted as inpatients via the ED during their study observation period. Of these patients who had an ED-related hospitalization, a total of 150 patients (13\%) underwent an

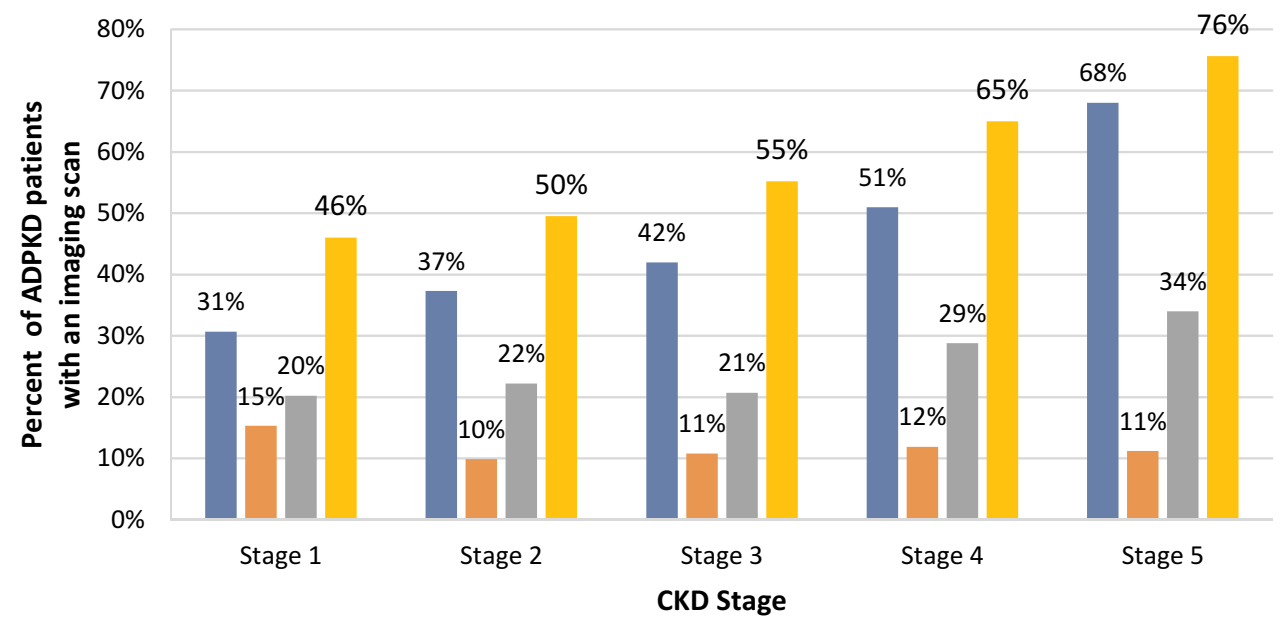

Figure 2 Proportion of ADPKD patients with imaging (CT, MRI, or ultrasound) during the observation period by CKD stage $(n=2,717)$.

Note: $* * * p<0.0001$.

Abbreviations: ADPKD, autosomal dominant polycystic kidney disease; CKD, chronic kidney disease; CT, computed tomography; MRI, magnetic resonance imaging. 
abdominal imaging scan as part of their inpatient hospitalization; nearly all received a $\mathrm{CT}$ scan. None of these patients received an MRI scan during their ED-related hospitalization (Table 3).

\section{Discussion}

This study provides important insight into real-world utilization patterns of imaging scans in patients diagnosed with ADPKD, a critical process for risk stratification. However, it is important to note that not all imaging is performed for the purpose of ADPKD diagnosis or risk stratification. Consequently, some imaging scans in this sample may have been performed for assessment of kidney volumes, to rule out an acute ADPKD-related renal condition (eg, infection, pain, cyst rupture, or cyst hemorrhage), or to evaluate a condition unrelated to ADPKD. Further, imaging of patients in this study that took place in the ED and during hospitalization was most likely performed for complications or incidental problems of ADPKD, not for risk stratification of ADPKD.

This retrospective database analysis found that among patients with ADPKD, roughly half received at least one abdominal scan during the observation period. The study also found that within this subgroup of patients who received at least one scan, patients underwent CT scans far more often than MRI scans or ultrasounds - a pattern that continued across CKD stage and across health care setting. Recommendations from the 2015 Kidney Disease: Improving Global Outcomes Controversies Conference on ADPKD do not include guidance as to the ideal frequency of abdominal imaging for monitoring, instead suggesting that imaging should occur on initial evaluation and is appropriate for at-risk patients presenting with medical complications (eg, hypertension, hematuria). ${ }^{20}$ Clinicians could benefit from resources that standardize frequency of imaging to assess disease progression.

This study found that the use of abdominal imaging scans, in general, was relatively low across health care settings over the observation period. While roughly half of the patients seen in an outpatient setting received an imaging scan during their observation period, the rate of imaging scans was much lower in all other health care settings. Although the patients in this study may have undergone an imaging scan before the study period to establish the ADPKD diagnosis, these modest rates suggest that many patients are not routinely assessed for the rate of disease progression and, therefore, ADPKD treatment may not be considered early enough to delay the onset of ESRD. Further, because Mayo class imaging classification tends to remain consistent over time, patients classified as low risk (ie, class 1A) generally would not undergo subsequent imaging. Patients in the current study who had their first imaging performed before the ascertainment period (ie, 2014-2017) may not have needed to undergo additional imaging during the study. However, this was likely not a widespread occurrence in our study population given that the Mayo class was published in 2015.

These low rates of imaging scans were also surprising and notable given more recent guidance about the importance of imaging in risk stratification and for diagnosis in individuals at risk of ADPKD. ${ }^{14-16}$ This finding may suggest that imaging-based risk stratification may be difficult to implement in real-world ADPKD clinical practice patterns. Imaging techniques — such as digital retinal imaging to identify microvascular pathology or diffusion-weighted imaging - could be informative for identifying the degree of renal impairment and for risk stratification in early CKD stages in particular. ${ }^{21-23}$ Conversely, our findings demonstrate that more imaging is performed in later stages of renal disease; this is problematic because ADPKD symptoms are present across all stages, not just later in the disease process. Low-dose or ultra-low dose CT protocols may perform as well as MRI in capturing total kidney volume, making these approaches potential risk stratification tools in clinical settings with limited resources or time. Further, total kidney volume is a more accurate measure of renal impairment than eGFR, which can capture disease impairment too late when used as the sole prognostic tool. ${ }^{24}$

Gradzik and colleagues recently called for diagnostic imaging to be the standard of care when diagnosing individuals with at least a $50 \%$ risk of ADPKD (offspring, siblings). ${ }^{15}$ And the Canadian Expert Consensus on Assessing Risk of Disease Progression and Pharmacological Management of Autosomal Dominant Polycystic Kidney Disease recently advised that all patients with a diagnosis of ADPKD or suspected ADPKD be referred to a nephrologist for an initial assessment that includes kidney imaging and, in some cases, genetic testing. ${ }^{25}$ However, imaging scans may not be needed in all cases. In limited-resource settings, it may be possible to use ultrasound-measured kidney length as a prognostic indicator of future renal disease instead of CT or MRI. ${ }^{26}$ Although this analysis used a kidney length of $16 \mathrm{~cm}$, patients with large kidneys (ie, $>20 \mathrm{~cm}$ ) are likely 
to have a large $\mathrm{TKV}$, and higher resolution imaging may be unnecessary.

The low rates of MRI scans across all health care settings and all CKD stages are noteworthy given that some experts consider MRI to be the "gold standard" modality for estimating TKV and that MRI is the imaging modality used in major longitudinal observational studies of ADPKD. ${ }^{15}$ Although the proportion of patients receiving any radiographic imaging scan was greater with disease severity (CKD stage), the proportion receiving MRI scans varied little by CKD stage. This observation was surprising given that MRIs have been highlighted as a highly effective and accurate tool in assessing progression of ADPKD disease. ${ }^{14,27}$ This pattern of less-frequent MRI use relative to $\mathrm{CT}$ imaging was consistent across all stages of disease severity and all health care settings and may warrant further research to determine whether utilization differences stem from clinical need or from other systematic barriers, such as lack of access, lack of reimbursement, or lack of understanding of the value of MRI in managing ADPKD.

\section{Limitations}

The findings in this study are subject to limitations associated with administrative medical claims data. These data are compiled for reimbursement purposes and not research, and thus the validity of the data diagnosis codes is uncertain. A diagnostic code may reflect either a confirmed diagnosis or a rule-out diagnosis, potentially causing an overcount of diagnosed (eg, comorbid) conditions. Different treatment settings may bill for services differently depending on differences in provider contracting and ownership. In some health care settings, a single imaging event may be billed with both a facility and a professional (eg, the radiologist and the radiology facility) claim, while in other settings (eg, the radiologist is an employee of the facility), such an event may be billed with only a facility claim. While this study assumes claims with identical service dates represent the same scan episode, contracting and ownership differences may affect counts when radiologist (professional) services are billed for a date later than the facility claim. In addition, claims data do not include the indication for a service or procedure. Regardless, administrative claims data provide necessary insight into real-world treatment patterns, especially for rare conditions such as ADPKD. Finally, this analysis examined imaging that patients underwent only during the observation period. The optimal periodicity for imaging in ADPKD, a relatively slow-progressing disease, has not been determined for estimating progression rate. Obtaining scans every several years may have been deemed acceptable in the past when no effective treatment was available.

\section{Conclusions}

ADPKD can be a resource-intensive and even lifethreatening condition as it progresses to ESRD. Risk stratification, whereby patients with rapidly progressing ADPKD are identified and prioritized for treatment, is an important component of ADPKD management. This study suggests possible underuse of abdominal imaging scans in patients with ADPKD across different health care settings for risk stratification, and possible underuse of MRI, which is the most accurate modality for estimating disease progression and progression risk. As kidney volumes may be more useful for risk stratification when renal function is preserved, all patients with CKD stages 1 and 2 at least should have abdominal imaging performed. However, these findings do not provide sufficient insight into why providers may be underutilizing imaging scans for their patients with ADPKD. Future research could help clarify whether less frequent utilization may stem from limited access to imaging services, insufficient reimbursement for these services, or a lack of understanding of the value of imaging services for patients with ADPKD. These findings may help various stakeholders (providers, patients, payers, policymakers) broaden access to imaging services that may facilitate risk stratification within this population, ensuring that patients at greatest risk of ESRD receive treatment that slows disease progression, maintains quality of life, and minimizes health care expenditures.

\section{Abbreviations}

ADPKD, autosomal dominant polycystic kidney disease; angio, angiography; CKD, chronic kidney disease; CPT-4, current procedural terminology, fourth edition; CRISP, Consortium for Radiologic Imaging Studies of Polycystic Kidney Disease; CT, computed tomography; ED, emergency department; eGFR, estimated glomerular filtration rate; ESRD, end stage renal disease; FDA, US Food and Drug Administration; GFR, glomerular filtration rate; htTKV, height-adjusted total kidney volume; ICD-9-CM, International Classification of Diseases, Ninth Revision, Clinical Modification; MRI, magnetic resonance imaging; REPRISE, Replicating Evidence of Preserved Renal Function: An Investigation of Tolvaptan Safety and 
Efficacy in ADPKD; SD, standard deviation; TEMPO, Tolvaptan Efficacy and Safety in Management of Autosomal Dominant Polycystic Kidney Disease and Its Outcomes; U/S, ultrasound; US, United States; w/, with; w/o, without.

\section{Ethics Approval and Informed Consent}

All data were fully de-identified to protect patient confidentiality (eg, no names, addresses, social security or medical record numbers, or other obvious identifiers) and could not be linked to identifiable information. Informed consent was not required.

\section{Acknowledgments}

Lisa Baker, $\mathrm{PhD}$, and Emily Kuhl, $\mathrm{PhD}$, of Global Outcomes group provided writing support and Esther Tazartes, MS, of Global Outcomes Group provided editorial support. This study, as well as editorial support, was funded by Otsuka Pharmaceutical Development \& Commercialization, Inc.

\section{Author Contributions}

All authors have met the IMCJE recommendations for authorship credit and contributed to the conception, study design, execution, acquisition of data, analysis and interpretation, or write-up of this study, or some combination thereof. All authors made substantial contributions to conception and design, acquisition of data, or analysis and interpretation of data; took part in drafting the article or revising it critically for important intellectual content; agreed to submit to the current journal; gave final approval of the version to be published; and agree to be accountable for all aspects of the work.

\section{Disclosure}

Dr Seliger received grant funding from Reata Pharmaceuticals, Kadmon Corporation, Palladio Biosciences, Otsuka Pharmaceuticals, and Sanofi. Ms Aigbogun and Ms Pao are former employees of Otsuka Pharmaceutical Development \& Commercialization, Inc., and Mr Stellhorn is an employee of Otsuka Pharmaceutical Development \& Commercialization, Inc. Christina S. Pao is currently an employee of GSK, and Myrlene Sanon Aigbogun is currently an employee of The Janssen Pharmaceutical Companies of Johnson \& Johnson. The authors report no other conflicts of interest in this work.

\section{References}

1. National Institute of Diabetes and Digestive and Kidney Diseases. Polycystic Kidney Dis. 2007.

2. Gabow PA. Autosomal dominant polycystic kidney disease. Am J Kidney Dis. 1993;22:511-512. doi:10.1016/S0272-6386(12)80921-8

3. Levy M, Feingold J. Estimating prevalence in single-gene kidney diseases progressing to renal failure. Kidney Int. 2000;58:925-943. doi:10.1046/j.1523-1755.2000.00250.x

4. Willey C, Kamat S, Stellhorn R, Blais J. Analysis of nationwide data to determine the incidence and diagnosed prevalence of autosomal dominant polycystic kidney disease in the USA: 2013-2015. Kidney Dis. 2019;5(2):107-117. doi:10.1159/000494923

5. Grantham JJ. Autosomal dominant polycystic kidney disease. $N$ Engl J Med. 2008;359(14):1477-1485. doi:10.1056/NEJMcp0804458

6. Bergmann C, Guay-Woodford LM, Harris PC, Horie S, Peters DJM, Torres VE. Polycystic kidney disease. Nat Rev Dis Primers. 2018;4(1):50.

7. Irazabal MV, Rangel LJ, Bergstralh EJ, et al. CRISP Investigators. Imaging classification of autosomal dominant polycystic kidney disease: a simple model for selecting patients for clinical trials. $J \mathrm{Am}$ Soc Nephrol. 2015;26:160-172. doi:10.1681/ASN.2013101138

8. Chapman AB, Bost JE, Torres VE, et al. Kidney volume and functional outcomes in autosomal dominant polycystic kidney disease. Clin J Am Soc Nephrol. 2012;7:479-486. doi:10.2215/CJN.09500911

9. Perrone RD, Mouksassi M-S, Romero K, et al. Total kidney volume is a prognostic biomarker of renal function decline and progression to end-stage renal disease in patients with autosomal dominant polycystic kidney disease. Kidney Int Rep. 2017;2(3):442-450. doi:10.1016/j.ekir.2017.01.003

10. Torres VE, Chapman AB, Devuyst O, et al. TEMPO 3:4 Trial Investigators. Tolvaptan in patients with autosomal dominant polycystic kidney disease. $N$ Engl J Med. 2012;367(25):2407-2418. doi:10.1056/NEJMoa1205511

11. Torres VE, Chapman AB, Devuyst O, et al. REPRISE Trial Investigators. Tolvaptan in later-stage autosomal dominant polycystic kidney disease. $N$ Engl J Med. 2017;377(20):1930-1942. doi:10.1056/NEJMoa1710030

12. Otsuka Pharmaceutical Development \& Commercialization, Inc. Jynarque (tolvaptan) [drug approval package]. U.S. Food and Drug Administration. Available from: www.accessdata.fda.gov/drugsatfda docs/nda/2018/204441Orig1s000TOC.cfm. Accessed June 23, 2020.

13. Rahbari-Oskoui F, Mittal A, Mittal P, Chapman A. Renal relevant radiology: radiologic imaging in autosomal dominant polycystic kidney disease. Clin J Am Soc Nephrol. 2014;9:406-415. doi:10.2215/ CJN.08940813

14. Chebib FT, Perrone RD, Chapman AB, et al. A practical guide for treatment of rapidly progressive ADPKD with tolvaptan. $J$ Am Soc Nephrol. 2018;29(10):2458-2470. doi:10.1681/ASN.2018060590

15. Gradzik M, Niemczyk M, Gołębiowski M, Pączek L. Diagnostic imaging of autosomal dominant polycystic kidney disease. Pol J Radiol. 2016;81:441-453. doi:10.12659/PJR.894482

16. IBM Watson Health. IBM marketscan research databases for life science researchers; 2018. Available from: https://www.ibm.com/ downloads/cas/0NKLE57Y. Accessed April 2018.

17. 2018 United States Renal Data System (USRDS). USRDS Annual Data Report, Volume 1: CKD in the United States. Chapter 2. Identification and care of patients with CKD. Available from: https://www.usrds.org/annual-data-report/previous-adrs/. Accessed February 10, 2020.

18. Quan H, Sundararajan V, Halfon $\mathrm{P}$, et al. Coding algorithms for defining comorbidities in ICD-9-CM and ICD-10 administrative data. Med Care. 2005;43(11):1130-1139. doi:10.1097/01.mlr.0000182534.19832.83

19. Charlson ME, Pompei P, Alex KL, et al. A new method of classifying prognostic comorbidity in longitudinal studies: development and validation. J Chronic Dis. 1987;40(5):373-383. doi:10.1016/00219681(87)90171-8 
20. Chapman AB, Devuyst O, Eckardt KU, et al. Autosomal-dominant polycystic kidney disease (ADPKD): executive summary from a Kidney Disease: Improving Global Outcomes (KDIGO) Controversies Conference. Kidney Int. 2015;88(1):17-27. doi:10.1038/ki.2015.59

21. Kannenkeril D, Frost S, Nolde JM, et al. Microvascular changes at different stages of chronic kidney disease. J Clin Hypertens. 2020.

22. Yeung L, Wu IW, Sun CC, et al. Early retinal microvascular abnormalities in patients with chronic kidney disease. Microcirculation. 2019;26(7):e12555. doi:10.1111/micc. 12555

23. Liu H, Zhou Z, Li X, et al. Diffusion-weighted imaging for staging chronic kidney disease: a meta-analysis. Br J Radiol. 2018;91 (1091):20170952. doi:10.1259/bjr.20170952

24. Bevilacqua MU, Hague CJ, Romann A, et al. CT of kidney volume in autosomal dominant polycystic kidney disease: accuracy, reproducibility, and radiation dose. Radiology. 2019;291(3):660-667. doi:10.1148/radiol.2019181830
25. Soroka S, Alam A, Bevilacqua M, et al. Updated Canadian expert consensus on assessing risk of disease progression and pharmacological management of autosomal dominant polycystic kidney disease. Can J Kidney Health Dis. 2018;5:2054358118801589. doi:10.1177/ 2054358118801589

26. Bhutani H, Smith V, Rahbari-Oskoui F, et al. A comparison of ultrasound and magnetic resonance imaging shows that kidney length predicts chronic kidney disease in autosomal dominant polycystic kidney disease. Kidney Int. 2015;88(1):146-151. doi:10.1038/ ki.2015.71

27. King BF, Torres VE, Brummer ME, et al. Consortium for radiologic imaging studies of polycystic kidney disease (CRISP). Magnetic resonance measurements of renal blood flow as a marker of disease severity in autosomal-dominant polycystic kidney disease. Kidney Int. 2003;64:2214-2221. doi:10.1046/j.1523-1755.2003.00326.x
The International Journal of Nephrology and Renovascular Disease is an international, peer-reviewed open-access journal focusing on the pathophysiology of the kidney and vascular supply. Epidemiology, screening, diagnosis, and treatment interventions are covered as well as basic science, biochemical and immunological studies. The manuscript management system is completely online and includes a very quick and fair peer-review system, which is all easy to use. Visit http://www.dovepress.com/testimonials.php to read real quotes from published authors. 